\title{
La escuela colombiana: entre la nuda vida y la biopotencia*
}

Colombian Schooling-Between Bare Life and Biopotency

A escola colombiana: entre a nuda vida e a biopotência

\author{
Amanda Cortés Salcedo** iD orcid.org/0000-0002-8579-3091 \\ Tito Hernando Pérez *** (iD) orcid.org/0000-0002-3377-9108 \\ Luis Francisco Guerra**** (iD) orcid.org/0000-0002-1475-9363
}

Artículo de investigación

Revista Colombiana de Educación, N.71. Segundo semestre de 2016, Bogotá, Colombia.

Para citar: Cortés, A., Pérez, T., y Guerra, F. (2O16). La escuela colombiana: entre la nuda vida y la biopotencia. Revista Colombiana de Educación, (71), 383-397.

Recibido: 21/02/16

Evaluado: $22 / 03 / 16$

* Parte de este escrito fue presentado como ponencia en la Primera Bienal de Infancias y Juventudes realizada en noviembre de 2013 . El tema abordado corresponde a avances de la investigación "Escuela y conflicto armado en Colombia", financiada por la Universidad Santo Tomás.

** Doctora en Educación de la Universidad Pedagógica Nacional. Docente e investigadora de la Maestría en Educación de la Universidad Santo Tomás. Correo electrónico: amandacortes@ustadistancia.edu.co

*** Candidato a Doctor en Estudios Sociales de América Latina en la Universidad de Córdoba, Argentina. Magister en Docencia de la Universidad de la Salle. Docente, investigador de la Maestría en Educación de la Universidad Santo Tomás.Coordinador de Investigaciones. Correo electrónico: tito.perez@ustadistancia.edu.co

**** Magíster Investigación Social Interdisciplinaria de la Universidad Distrital Francisco José de Caldas Docente, investigador de la Maestría en Educación de la Universidad Santo Tomas. Coordinador línea educación y derechos humanos.. Correo electrónico: luis.guerra@ustadistancia.edu.co 


\section{Resumen}

Leer la relación entre escuela y conflicto armado resulta una tarea retadora pues sería dificil no caer en la tentación de afirmar, casi de inmediato, que las políticas han fracasado. Esta afirmación sería ampliamente demostrable a partir de un robusto corpus documental de informes presentados por organismos internacionales, por la sociedad civil o por las mismas instituciones del Estado colombiano que han mostrado datos cada vez más alarmantes sobre situaciones violatorias de derechos humanos.

El reclutamiento forzoso de niños y jóvenes les priva de su derecho a la educación y vulnera su integridad física y emocional; las amenazas a maestros en zonas de confrontación vulnera su derecho al trabajo, a la libre expresión, a la libertad de cátedra y a la vida misma; y las escuelas que funcionan en estas zonas son usadas por los distintos actores del conflicto en una clara violación al derecho internacional humanitario.

Todas estas situaciones, sin duda, han tenido efectos en cómo se configura la institución escolar para operar en medio del conflicto armado, también en la subjetividad del sujeto escolar y del sujeto docente que no tienen otra opción que permanecer en estos territorios.

Así, la pregunta es ¿cómo se resisten estas escuelas a las políticas de muerte y se recrean en circunstancias de excepción?

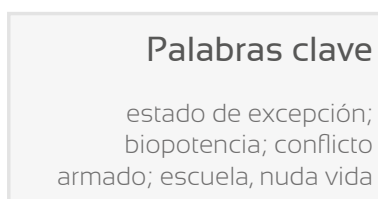

Abstract

Reading the school-armed conflict relationship is a challenging task. It would be difficult not to fall into the temptation to say, almost immediately, that policies have failed. Such a statement would be widely demonstrable from a robust corpus of documentary reports by international organizations, civil society or by the same Colombian State institutions, which have shown increasingly alarming data about human rights violations.

Forced recruitment of children and young deprives them of their right to education and undermines their physical and emotional integrity. Threats to teachers in areas of confrontation violates their right to work, to free speech, academic freedom and life itself. Schools operating in these areas are used by actors in the conflict, which constitutes a clear violation of international humanitarian law.

All these situations have certainly had an impact on how the school is set to operate in the midst of armed conflict, as well as in the subjectivity of the school subject and subject teachers who have no other choice than to stay in these territories

So, the question is: How do these schools resist the policies of death, and recreate themselves under exceptional circumstances?

\section{Resumo}

Ler a relação entre escola e conflito armado é uma tarefa desafiante, pois poderia ser dificil não cair nas tentações de afirmar, quase imediatamente, que as políticas têm fracassado. Este afirmação poderia ser completamente demonstrável desde um grande corpus documental de informes apresentados pelos organismos internacionais, pela sociedade civil ou pelas mesmas instituições do Estado colombiano que manifestaram dados cada vez mais alarmantes sobre as situações violadoras dos direitos humanos.

O recrutamento forçado das crianças e jovens privá-los do seu direito á educação e afeta seu integridade física e emocionai; as ameaças á professores em áreas de confronto, afeta seu direito ao trabalho, á liberdade de expressão, á liberdade de cátedra y á vida mesma; y as escolas que trabalham nestas áreas são usadas pelos diferentes atores do conflito numa clara violação á direito internacional humanitário

Todas estas situações, sim divida, tem tido efeitos em como si configuram a instituição escolar para trabalhar no meio do conflito armado, também na subjetividade do sujeito escolar e do sujeito docente que não tem outra opção mais que ficar nestes territórios. 
Hay siempre algo, en el cuerpo social, en las clases, grupos, en

los propios individuos, que se escapa de una cierta manera a

las relaciones de poder; algo que no es la materia prima más o menos dócil u obstinado, sino que es el movimiento centrífugo,

la energía inversa, la fuga

Michel Foucault

\section{Introducción}

U

n robusto corpus documental de informes presentados por organismos internacionales, por la sociedad civil y por las mismas instituciones del Estado colombiano muestra datos cada vez más alarmantes sobre situaciones violatorias de los derechos de niños y jóvenes en Colombia. El aumento del desplazamiento forzado de este sector de la población, del reclutamiento forzado en las filas de los grupos armados, del asesinato, el secuestro y la violencia sexual ${ }^{1}$ son situaciones que, sin duda, han operado en el sujeto niño/joven, y los han constituido de manera distinta. Esto es lo que hemos Ilamado efectos de subjetivación (Cortés, 2013).

Por otro lado, las discusiones sobre la escuela parecieron agotarse en la disputa intelectual de los 90, que la anunció bien como el "primer espacio público democrático" en el que el niño se forma y por tanto es allí donde se aprende a ser ciudadano; o bien como la institución en la que el alumno es un sujeto producido por tecnologías disciplinarias.

Hoy, desde una u otra orilla, los análisis parecen desembocar en discursos prescriptivos o en diagnósticos desalentadores, lo cual parece no dejar mucho margen para nuevos análisis desde esas perspectivas. Lo que se propone en primera instancia es que, en lugar de aceptar el "fracaso" de esas políticas, pensáramos que estamos frente a prácticas de gobierno ${ }^{2}$ de esa población en circunstancias de excepción $n^{3}$ en las que la línea entre la protección de su vida y la promoción de su muerte (física o simbólica) comienza a ser cada vez más tenue.

En segundo lugar, comprender la emergencia de la escuela contemporánea colombiana (Cortés, 2012) como una institución en la que operan políticas de muerte que coinciden con el reconocimiento de las múltiples violencias en el país y el reconocimiento del conflicto armado, provocó que todos los esfuerzos se enfilaran a contrarrestar el efecto negativo de una escuela autoritaria, antidemocrática pero sobre todo violentadora de los derechos humanos. Sin embargo, la historia reciente de la educación

1 Ver informes Unesco, Coalico, Unicef, Corporación Nuevo Arcoíris, War Child, entre otros.

2 Entendido el gobierno como la conducción de las conductas (cfr. Foucault, 2008).

3 La noción de excepción se tomó de Giorgio Agamben y se explicará más adelante.

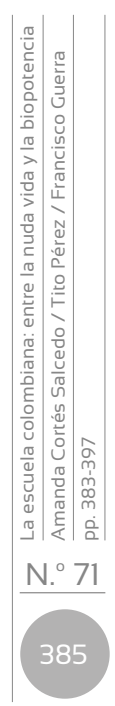


también da cuenta de cómo las prácticas de muchos maestros desafían el destino de la escuela en territorios de guerra.

Por estas razones nos hemos preguntado ¿Cómo se administra la vida de quienes habitan la escuela en situación de excepción, provocada para nuestro caso por el conflicto armado? ¿Cómo resisten estas escuelas a las políticas de muerte y se recrean en y por la vida? Para acercarse a las posibles respuestas se hizo necesario construir unos referentes que posibilitaran una lectura menos pesimista y no tan optimista; es decir que se tomaron precauciones frente a la tentación de diagnosticar o idealizar.

El propósito ha sido el de realizar una lectura más antropológica, que reconozca en las voces de quienes viven el día a día de la guerra un saber legítimo que interpele cualquier prescripción política o científica.

De esta manera el lector irá descubriendo en esas mismas voces de qué manera hemos pasado de unas políticas de la vida a unas sobre la vida en las que maestros y estudiantes transitan como homos sacer en la nuda vida, la escuela se convierte en un campo y la potencia de-vida se abre paso.

\section{Maestros y estudiantes: entre la nuda vida y el Homo sacer}

Para arriesgar posibles comprensiones sobre cómo ha sido administrada la vida de quienes habitan la escuela en situación de excepción, provocada para nuestro caso por el conflicto armado, es necesario pensar en la vida y en lo que la constituye.

Las prácticas de biopolítica ${ }^{4}$ y de soberanía, estudiadas inicialmente por Michel Foucault, pueden verse como una sucesión cronológica. En las primeras se inscriben la libertad y la resistencia al igual que todos los mecanismos que producen la vida misma y, a su vez, la vuelven productiva; este es el biopoder. Para el filósofo francés, la biopolítica remite al modo en que la vida biológica de la población en su conjunto se ha convertido en objeto de administración y gobierno mediante los mecanismos de normalización ${ }^{5}$, que no funcionan del mismo modo que los dispositivos jurídicos de la ley.

4 El filósofo colombiano, Rubén Sánchez, señala que tanto biopolítica como biopoder son usadas por Foucault, para diferenciar entre una forma de racionalidad política y un conjunto de tecnologías de gobierno desplegadas por ella. "En efecto en tanto que la tecnología biopolítica puede ser definida como el conjunto de procedimientos gracias a los cuales se produce una población, el biopoder puede ser considerado como una forma de racionalidad política que somete a la vida produciendo a partir de ella individuos y poblaciones" (Sánchez, 2007, p. 34).

5 La normalización, desde el modelo disciplinario, se construye a partir de un determinado resultado que se consigue siempre y cuando los actos de la gente se ajusten a una medida 'normal'. Por el contrario, en los dispositivos de seguridad, la normalización opera no desde la prescripción, ni del impedir que algo suceda, sino que desde una racionalidad basada en la probabilidad y el azar, se deja actuar interviniendo la población desde el cálculo del riesgo, la peligrosidad y la individualización del fenómeno colectivo. 
Un autor posterior a Foucault, que lo retoma para distanciarse de él, es Agamben (1998), quien define la biopolítica como una serie de dispositivos mediante los cuales el ejercicio de la soberanía estatal transforma la vida humana, individual o colectiva, en nuda vida (vida desnuda), es decir, expuesta a la muerte.

Los dos autores coinciden en suponer que es en la soberanía en la que opera el poder sobre la vida y no el poder de producir la vida. Al respecto podríamos decir que en el poder biopolítico sería inadmisible el poder soberano, pues no podría producirse la vida y al mismo tiempo exterminarla; sin embargo los dos también pueden aparecer como una superposición de contraste, casi paradójico.

Este contraste puede leerse en las preguntas que plantea Foucault:

¿Cómo es posible que un poder político mate, reclame la muerte, la demande, haga matar, dé la orden de hacerlo, exponga a la muerte no solo a sus enemigos sino aun a sus propios ciudadanos? ¿Cómo puede dejar morir ese poder, que tiene el objetivo esencial de hacer vivir? ¿Cómo ejercer la función de la muerte, en un sistema político centrado en el biopoder? (2000, p. 230).

El autor ve entonces no una disrupción sino una completud, aludiendo a un proceso de mutación paulatina en la que el modelo soberano incorpora en sí mismo el antiguo poder pastoral y el modelo biopolítico recoge algo del poder soberano. En este punto Michel Foucault sitúa lo que denominó racismo.

En efecto, ¿qué es el racismo? En primer lugar, el medio de introducir un corte en el ámbito de la vida que el poder tomó a su cargo: el corte entre lo que debe vivir y lo que debe morir [...]. Por otro lado, el racismo tendrá su segunda función: su papel consistirá en permitir establecer una relación positiva, por decirlo así, del tipo "cuanto más mates, más harás morir", o "cuanto más dejes morir, más, por eso mismo, vivirás". Pero el racismo, justamente, pone en funcionamiento, en juego, esta relación de tipo guerrero - "si quieres vivir, es preciso que el otro muera" ${ }^{\prime}$, de una manera que es completamente novedosa y decididamente compatible con el ejercicio del biopoder. [...] La importancia del racismo en el ejercicio de un poder semejante es la condición gracias a la cual se puede ejercer el derecho de matar. Desde luego, cuando hablo de dar muerte no me refiero simplemente al asesinato directo, sino también a todo lo que puede ser asesinato indirecto: el hecho de exponer a la muerte, multiplicar el riesgo de muerte de algunos o, sencillamente, la muerte política, la expulsión, el rechazo, etcétera [...]. En líneas generales, creo que el racismo atiende la función de muerte en la economía del biopoder (2000, p. 231).

El racismo como constructor de otredad que se vincula al peligro y riesgo de la propia vida -la individual y la colectiva- es un elemento 
de la biopolítica en el que se expresa en la máxima "hacer vivir, dejar morir". Dos acciones que prefiguran un sentido positivo y otro negativo de las políticas de la vida; la primera, la del hacer vivir, produjo una serie de operaciones sobre la población para regularla como especie, su reproducción, su salud, enfermedad, productividad, etcétera. La segunda, dejar morir, constituye en estricto sentido la tanatopolítica. Esta es una tecnología específica del poder moderno que no tiene siempre como fin la eliminación física de las otredades, sino el disciplinamiento social del conjunto poblacional.

Así, las políticas de muerte no son un fin sino un medio para gobernar la población a través de mecanismos como el miedo. La guerra puede ser considerada también un medio pero no solamente por el control de territorios o de eliminación directa del enemigo, sino de la producción de formas de comportarse que se necesitan funcionales a una racionalidad política, en este caso lo que varios autores denominan tanatopolítica (Agamben, 1998; Foucault, 2000) o necropolítica (Mbembe, 2011).

Por su parte la noción de nuda vida Agamben la sitúa originalmente al margen del orden jurídico pero afirma que coincide de "manera progresiva con el espacio político, de forma que exclusión e inclusión, externo e interno, bios y zoe, derecho y hecho, entran es en una zona irreductible de indiferenciación" (1998, p. 19). La zoe es el término griego que aludía al simple hecho de vivir, común a todos los seres vivos; es el sujeto privado, mientras que el bios se referirá a una forma de vivir propia de determinado grupo, es el sujeto político.

La zoe es la nuda vida, una vida que se posesiona en el umbral entre lo humano y lo no humano, incluida por exclusión, construida a partir de dos figuras del derecho arcaico: el Homo sacer y el bando. Según explica Agamben, el Homo sacer refiere a una figura del derecho romano que se aplica a quienes, por haber cometido un delito, se les podía dar muerte sin que eso implicase homicidio. El bando por su parte se refiere a una figura del derecho germánico que designaba tanto la exclusión de una persona de la comunidad como el mandato y la enseñanza del soberano. Pero el que ha sido puesto en bando no queda sencillamente fuera de la ley ni es indiferente a esta, sino que es abandonado por ella. Es decir que queda expuesto y en peligro en el umbral en que vive.

Los sujetos que habitan aquellas escuelas que se encuentran entre el fuego cruzado son resultado de ese racismo pues han sido situados en el margen de su condición de ciudadanos, en una clara forma de disciplinamiento y de control sobre su estatus político desde lo que hemos llamado unas políticas de muerte.

Criscione (2011) analiza este tipo de políticas en un periodo de gobierno de la historia reciente de Colombia y diferencia dos tipologías 
según los efectos de las prácticas que se definen a partir de su funcionamiento (prácticas físicas, discursivas y simbólicas).

De manera resumida, el contundente aporte de este autor distingue prácticas no directamente homicidas, como la tortura y la detención arbitraria, que tienen efectos de disciplinamiento social sobre sus víctimas, y que funcionan en el plano físico y psicológico con el dolor y el miedo y en el plano discursivo con prácticas como la estigmatización, la delación y la amenaza. Se pretende "corregir", "recuperar" y normalizar al que se considera "peligroso".

Las prácticas homicidas, como las ejecuciones extrajudiciales ("falsos positivos" y "limpieza social"), tienen un efecto directo sobre el cuerpo de la víctima pues se busca su aniquilamiento, que no es necesariamente físico sino que puede ser simbólico y convertir a las víctimas en bandos $u$ Homo sacer, reduciendo su bios a simple zoe. Es el excedente de la población que debe desecharse bien en fosas comunes, bien en desplazados que como nómadas transitan entre el retorno y las diásporas constantes abandonados a su suerte.

Criscione establece así la hipótesis de la triple funcionalidad de las prácticas tanatopolíticas:

en primer lugar el aniquilamiento físico del sujeto peligroso o anormal. En segundo lugar, el disciplinamiento del cuerpo de los individuos que sufren directamente la práctica no directamente homicida. Y por último la normalización de la población a través del mecanismo del miedo que se establece partir de la implementación generalizada y sistemática de la muerte (2011, p. 25).

Esta clasificación del autor nos sirve para describir en el plano del micropoder cómo han operado las políticas de muerte en la escuela y en los sujetos que la habitan usando la guerra como medio ${ }^{6}$.

Las prácticas de aniquilamiento se evidencian en los homicidios de maestros, que suman alrededor de la tercera parte dentro de los asesinatos de sindicalistas. Según las fuentes consultadas, de 51 sindicalistas asesinados en el 2010, 29 eran maestros, y en el 2011, de 26 sindicalistas asesinados 14 eran maestros (Merizalde, 2012). Investigaciones develan en los relatos de los niños lo que han vivido por los combates que cercan sus escuelas y el horror de ser testigos de los asesinatos de sus maestros, como ocurrió en el municipio de la Hormiga, un claro ejercicio de disciplinamiento social y normalización mediante la instauración del temor a partir del "ejemplo", que deja un radical mensaje en los niños que observan los homicidios: "quien no está conmigo, está contra mí". Esto significa morir.

6 Se analizaron fuentes documentales como periódicos de amplia circulación, artículos de revistas y documentos de política educativa. 
Las amenazas a estudiantes se hacen de manera directa, los distintos actores armados asumen la escuela como un espacio potencial para el reclutamiento pero también como espacio peligroso donde se gesta el "enemigo". Así, la vigilancia sobre lo que les enseñan los maestros a los escolares es una práctica constante mediante las preguntas directas a estos y la observación directa en el aula, en donde se presentan armados, lo cual genera un efecto de intimidación y miedo.

Otras amenazas dirigidas a los estudiantes son las Ilamadas "pintas" que aparecen en las paredes de las casas en los barrios donde residen y generalmente visibles a la escuela. La famosa advertencia que se leía en muchos barrios pregonaba "los niños buenos se acuestan temprano, los malos los acostamos nosotros" se basa en la violación de las normas que han impuesto a punta de bala: "no robarás, no cometerás actos impuros, no codiciarás los bienes ajenos" (El Espectador, 2008). También a través de comunicados anónimos exigen cerrar las maquinitas de las tiendas a las ocho de la noche, o imponen toque de queda. Pero realmente las masacres de adolescentes y jóvenes, como prácticas homicidas, tienen un trasfondo que no es el simple incumplimiento de las normas, hay una economía de estas políticas de muerte que es evidente en lo que señala un líder comunitario "Si estos jóvenes que tienen problemas están identificados, ¿por qué no los inscriben en un proceso de resocialización? La respuesta es simple. Es más barato matarlos" (El Espectador, 2008).

El asunto es que en estas prácticas no solo participan las fuerzas paraestatales sino que se ha demostrado que las mismas fuerzas del Estado e inclusive la comunidad en la que habitan los jóvenes están involucradas en lo que se conoce como "limpieza social". Y en ella hay una justificación basada en el supuesto de que estos adolescentes son "caso perdido", acusados de ser violadores, drogadictos o ladrones, se aprueba directa o indirectamente "eliminarlos" para "hacer más seguro el barrio".

Las amenazas a los maestros, como prácticas no homicidas, tienen una función de aniquilamiento y de normalización más que de disciplinamiento. La soberanía de la ley en un Estado en situación de excepción debe calcular cómo proceder y nombra a quien está en exposición de muerte como "víctima", término que incorpora la condición de una vida nuda:

Se ha podido identificar que existen docentes y directivos docentes oficiales que, por diversas circunstancias, se encuentran bajo riesgo extraordinario o extremo, lo cual atenta contra sus derechos fundamentales como son: la vida, integridad, libertad y seguridad personal. Que en la aplicación de las medidas que adopte el Estado Colombiano para la protección de las personas referidas en el considerando anterior, se debe garantizar que no se afecte la unidad familiar, el derecho al trabajo y demás garantías constitucionales, con el fin de permitir que las víctimas tengan la posibilidad de continuar con su proyecto de vida (Decreto 1628 de 2012). 
El 27 de noviembre de 2013 en El Espectador, diario de circulación nacional, se afirmaba que 147 docentes del departamento de Córdoba habían sido amenazados y que esta situación la compartían 1117 maestros de todo el país.

Los casos de amenazas en las zonas urbanas, principalmente, son producidos por intimidaciones de padres de familia, estudiantes o pandillas juveniles, y en las zonas rurales, las amenazas provienen de las guerrillas y de los grupos armados ilegales post desmovilización de las Auc.

Las amenazas a docentes, en su mayoría, son de carácter extorsivo, a través de llamadas telefónicas de personas que se identifican como mandos de los grupos armados ilegales. En otros casos, las intimidaciones van acompañadas de panfletos. La Federación Colombiana de Educadores estima que desde 1995 a la fecha en Colombia se han registrado 1000 maestros asesinados, 4003 amenazados, 1092 desplazados, 60 desaparecidos y 70 refugiados.

Suárez (2014) en su columna, “¿Por qué se asesina a los maestros en Colombia?", señala que es por hacer política, así el despojamiento de su bios se reduce a la zoe, a la nuda vida pues como lo señala este historiador

para las fuerzas tradicionales regionales y para el paramilitarismo, la incursión de los maestros en la competencia electoral representa una amenaza a su hegemonía y control político, incluido el propósito de "refundar la patria". Por eso el periodo de mayor violencia contra los maestros, 1995-2001, corresponde al periodo de mayor expansión y criminalidad del paramilitarismo en el país (P. 10).

Nos atreveríamos a decir que es una tecnología que opera en la escuela, instalando lo que Restrepo (1999) Ilamó la "cultura de la muerte", en la que "la amenaza, es funcional para el establecimiento del orden, y en la que la protección de la vida está condicionada a la consumación cada vez más extendida de la muerte" (1999, p. 8). Reforzamos nuestro argumento siguiendo a Cayuela (2008), quien caracteriza la tanatopolítica como "un dispositivo de poder orientado a la guerra como forma de posibilidad política y ausencia absoluta de voluntad ciudadana en la toma de decisiones". Así que si en el escenario de guerra de muchas zonas de Colombia "la educación del ciudadano" parece impensable, no lo es suponer que la guerra opera desde la negación misma de la condición de ciudadano, como tecnología de gobierno.

Maestros y estudiantes son asumidos como efectos colaterales, como cómplices o como víctimas. Así los efectos de subjetivación van visibilizando esas formas de ser sujetos en la escuela; unos que tan solo 
existen, pues el temor les silencia hasta su misma posibilidad de ser; a otros, la indignación los lleva a trascender el mero estar en el mundo para ser con el mundo. Unos y otros en bando, es decir expuestos y arrojados a su suerte.

\section{De la escuela como campo}

En la investigación ha resultado útil tomar la noción de campo acuñada también por Agamben, quien lo precisa como el no lugar paradigmático de las políticas de exterminio. El campo se define como "el absoluto espacio biopolítico en el que el poder tiene en frente la pura vida biológica sin mediación alguna" (1998, p. 181). Para el filósofo italiano, el campo se abre cuando el estado de excepción empieza a convertirse en regla y cuando aquella suspensión temporal deviene constante, donde no hay protección jurídica alguna y el ciudadano se corresponde con el Homo sacer.

Para analizar los campos se hacen necesarios entonces los conceptos que propone de estado de excepción. La excepción es un fenómeno por el cual aquello que es excluido de la norma general no pierde relación con ello, sino que se mantiene relacionada en la forma de suspensión y el soberano es quien decide. El estado de excepción se generaliza cada vez más como tecnología del poder del Estado, bajo el paradigma de la seguridad en el que la situación provisional de peligro tiende a volverse regla y la excepcionalidad constante.

El estado de excepción se volvió una constante en Colombia y los campos proliferan. Uno de ellos es la escuela, específicamente la rural, donde la vida que se vive parece haber sido declarada como indigna de vivirse y donde el soberano decide a quién dejar vivir y a quién hacer morir. Maestros y estudiantes han sido incluidos en la exclusión, y su condición desnuda los arroja a la fosa de los desperdicios humanos, como los llama Bauman, a quienes se pueden aniquilar sin que ello implique homicidio.

Flor Romero (2013) muestra en su investigación que Colombia es uno de los países que registra mayor número de atentados contra el espacio escolar, verificable en ataques armados a las infraestructuras, minado de zonas adyacentes a los perímetros escolares, presencia de actores armados tanto regulares como no oficiales, uso del espacio escolar para el ejercicio propagandístico y de difusión de todo tipo de mensajes y de presión o aleccionamiento para las comunidades.

Recogiendo datos de distintos informes (Coalico, 2008; Fundación Dos Mundos, 2009 y Unesco, 2007), la investigadora caracteriza las arremetidas contra la escuela, que van desde ataques con explosivos y armas de fuego a la infraestructura hasta asesinatos selectivos, secuestro, 
detención ilegal, desaparición forzosa, tortura, reclutamiento forzado y violación sexual. En estas prácticas participan guerrilla, paramilitarismo y fuerzas militares.

Estas escuelas además han sido usadas "como sitio de alojamiento, como trincheras, como lugar para hacer proselitismo y reclutamiento, como centro de operaciones de tortura y entrenamiento, por parte de los actores armados" (p. 64). Pero también la escuela ha sido producida como espacio de protección, de creación y de cuidado.

\section{De las políticas de muerte a las prácticas de biopotencia}

La segunda pregunta que nos hicimos fue ¿Cómo la escuela resiste a las políticas de muerte y se recrea en y por la vida?

Los relatos que se recogieron en departamentos como Caquetá, Putumayo y Sincelejo nos permitieron ver que quienes en medio del conflicto armado no tienen otra opción que aprender a sobrevivir en él, sobreponen al poder sobre la vida el poder de la misma vida. Lo que narra un profesor de Caquetá, muestra cómo aparece cada día, en su recorrido hacía la escuela, ese fiero impulso de vivir ejerciendo su profesión.

Por causa del conflicto armado son muchas las minas antipersonales que hay en este territorio, personalmente trabajo en una escuela rural del municipio y lo que tengo claro es que durante el recorrido del camino no debo extraviarme ni cinco metros porque puedo quedar sin pies al caer en una mina antipersonal, son muchos los animales bovinos que han perdido su vida, también campesinos de la región han salido perjudicados por causa de las mismas. La zozobra que se vive es fatal, a veces he quedado al medio de los combates, tener que durar atrincherado hasta 4 horas para poder salvar mi vida y saber que a tan solo 100 metros de donde iba a pasar acaba de estallar muchas minas (Relato 1, 2011).

Ante la encarnación del deseo de muerte instalado en lo más cotidiano de la existencia de los escolares viene, no como reacción sino como fuerza inventiva, un artilugio pedagógico que va moviéndose rizomáticamente entre la pedagogía de los valores y las instrucciones para sobrevivir en medio del combate

Los niños desde muy pequeños mantienen ese deseo de tener una arma [sic] en las manos y en las diferentes instituciones hacen armas de palo y juegan al pistolero, todo esto porque observan personas armadas. En este caso lo profesores tienen un papel importante que es educar e inculcar valores que contrarresten el deseo de tener una arma en las manos, hay que explicar el daño que puede hacer una persona armada y por qué no deben usarlas ellos. Son los docentes los responsables 
de inculcar la necesidad en los niños de estudiar, son los docentes los que deben dar instrucciones al niño para no llegar a cometer un error en caso de una balacera o para no llegar a caer en un campo minado (Relato 1, 2011).

En el colegio $X$ ubicado en un lugar remoto del Departamento del Putumayo, un Rector y su grupo de docentes han decidido "no regalarle un joven más a la guerra". Esta institución sin "cercas" es tránsito de soldados del ejército y de paramilitares, pero el actor que más ejerce influencia es la guerrilla del frente 48 de las FARC. El control se hace sobre la capacidad de decidir, despojando de voluntad a quien se quiere sometido y es el mecanismo del miedo el que opera a partir de la imposición de una serie de normas ante la generación de sentimientos de impotencia y fragilidad

Ellos hacen un control del territorio, miran quién entra, quién sale en primer lugar y también en su forma de vida lo que está permitido y lo que no está permitido y también hay un control a la libre movilidad, entonces en cierto momento establecen horarios en los que uno se puede mover y horarios en los que uno no puede moverse. El derecho a la libre movilidad uno no lo puede ejercer, eso afecta digamos más que todo para hacer trabajos de investigación con los estudiantes o encuentros en ciertas horas de la tarde o noche no se pueden realizar y eso afecta la parte académica de los estudiantes y afectaría también un poco la calidad de vida, [...] ellos tienen un manual de convivencia donde hay unas ciertas cosas que permiten y ciertas cosas que prohíben y ciertas sanciones que ellos imponen. Desde la institución estamos hablando de nuestro manual de convivencia en el que se prioriza para nosotros el diálogo, mientras que lo que ellos priorizan es la sanción, entonces viene la represión. Esto no nos permite que se ejerza desde la escuela libremente y podamos avanzar más rápidamente en torno a la construcción de un escenario, desde la escuela, [para] establecer el diálogo como un camino para la resolución de conflictos (Relato 2, 2015).

A pesar del control intimidante del actor que gobierna no solo el territorio, sino las conductas de los habitantes, "'la vida' o aquello que parecía sometido, subsumido, controlado, dominado, revela en el proceso mismo de expropiación, su positividad indomable y primera" (Pál Pelbart, 2006, p. 9). Este rector, que ha nacido de las mismas entrañas de la selva putumayense, ha decidido asumir como un estilo de vida, como una estética de su existencia, no la reactividad al poder que se obstina en dominarle, sino la afirmación de su propia fuerza creadora.

Yo parto desde mi experiencia y desde mis convicciones, para decir no a la participación en el conflicto, siempre lo he tenido claro y nunca me he dejado, nunca he caído como en la trampa digamos, de estar haciéndole buena cara al uno y mala cara al otro para [poder] estar y mantenerse en el territorio. Yo más bien he sido claro con los profesores 
y respetando su pensamiento, sus tendencias, estoy mostrando un camino de que es lo que debemos ser y he trabajado mucho con ellos lo que es la neutralidad activa, es lo que más he impulsado dentro de los profesores, ser neutrales pero activos, que no indica ser pasivos, entonces, expresamos cada uno lo que sentimos lo que realmente queremos [...] es mejor sentar una posición frente a esto que se está viviendo. Que hemos tenido problemas, sí, en el 2013 tuvimos una situación donde la guerrilla sentó una posición en la cual no podíamos entrar en las veredas y posiblemente nos iban a llamar a que tuviéramos una reunión con ellos [...]. Nosotros nos reunimos y esperamos a que nos llamaran para sentar nuestra posición: que de ser así [de no poder entrar a las veredas], en bloque nos retirábamos (Relato 2, 2015).

Justamente es la decisión de hacer de la vida una obra de arte, como lo proponía Foucault, que las sensibilidades estéticas y morales se transforman en política como acción de-vida. Arte que nos Ileva a "la ilusión bella de anulación del sinsentido a través de la afirmación de un estilo propio", a la afirmación de la alegría por estar vivo, a crear, que no recrear, nuevas formas de habitar-se

Otro elemento que se ha trabajado ahí es con referencia a las escuelas, está la escuela de música, está la escuela de danza, está la escuela de deporte, está la escuela de teatro, que permite no solamente ocupar su tiempo libre. Lo que estamos buscando son espacios de participación de los muchachos donde puedan a través de la cultura, expresar lo que ellos hoy sienten, su parte cultural, pero también su realidad frente al conflicto que estamos viviendo y puedan tocar temas como el narcotráfico y como la misma violencia. Ya cuando están en noveno, decimo y once empezamos a hablar del proyecto de vida para tener en cuenta que esos elementos son reales y están dentro del territorio y que no podemos volver a la finca a cultivar nuevamente la coca, que tenemos que buscar otras alternativas de vida. Hemos tenido varias experiencias en donde a algunos jóvenes los hemos podido sacar de ese círculo vicioso del narcotráfico y la guerrilla y buscar otras alternativas. Es el caso de Iván, el caso de Jessica, son estudiantes que están en la Universidad de Lima. Ellos están terminando su profesión para volver a su territorio (Relato 3, 2015).

Solo las voces de quienes han logrado trazar esas imperceptibles pero estratégicas fugas a las mallas de un poder que se quiere opresor pueden dar testimonio de la potencia del bios, allí se constata que aún quedan intersticios en los que la práctica de libertad es posible, no obstante el miedo que produce la amenaza permanente, a pesar de ceñirse constantemente hasta sobre sus propias sombras la hoz que osa empuñar quien se ha autoproclamado soberano en territorios abandonados a su soledad cívica. 
Algunos docentes sienten mucho temor y sienten mucha incertidumbre dentro de la institución para ejercer libremente su derecho a la cátedra, otros docentes, aun con sus temores buscan estrategias pedagógicas que permitan a los estudiantes expresar y participar. Hemos trabajado en la parte curricular este tipo de actividades que permitan trabajar desde todas las áreas, que podemos expresar simpatía, pero no por las formas de violencia. Una cosa que se ha trabajado es la parte ética y moral, en algún momento mataron por ejemplo a tres personas, y uno de los estudiantes manifestó: ija! por algo les dieron. Eso fue motivo para que nos reuniéramos todos y habláramos sobre ese "por algo les dieron", estábamos avalando y comulgando una práctica violenta dentro del territorio. Hoy en día no he vuelto a escuchar eso y más bien desde la música he escuchado otro tipo de cosas que hablan en favor de la vida. Se le canta a la vida (Relato 4, 2015).

La escuela, por lo menos la de cada uno de estos maestros, puede ser un lugar de animación a la vida. Una escuela de juego, abierta, que resignifica la relación consigo mismo, con los otros y con el territorio, en una idea muy cercana a la idea filosófica de potencia, esa que tendrían los sujetos para relevar el dolor por el goce, expresar su voluntad para intervenir en la regulación de su propia vida y que le apuesta a construir una cultura de la vida para reemplazar la "cultura de la muerte". 


\section{Referencias bibliográficas}

Agamben, G. (1998). Homo sacer. El poder soberano y la vida nuda. Valencia: Pre-textos.

Cayuela, S. (2008). ¿Biopolítica otanatología? Revista deFilosofía Daimon, 43.

Cortés, A. (2012). Prácticas de ciudadanización en la escuela contemporánea. Colombia 1984-2004 (tesis doctoral). Universidad Pedagógica Nacional, Bogotá, Colombia.

Criscione, G. (2011). Las prácticas tanatopolíticas en los tiempos de la seguridad democrática (2002-2010) (tesis de maestría). Universidad Javeriana, Bogotá, Colombia.

Decreto 1628 de 2012. Por el cual se reglamenta parcialmente el artículo 22 de la Ley 715 de 2001, en relación con el procedimiento para la protección de docentes y directivos docentes que prestan sus servicios en los establecimientos educativos estatales ubicados en las entidades territoriales certificadas en educación que se encuentran bajo riesgo extraordinario o extremo.

En Colombia hay 1.117 maestros amenazados (27 de noviembre de 2013). El Espectador.

Es que es más barato matarlos. (5 de septiembre de 2008). El Espectador.

Foucault, M. (2000). Defender la sociedad. México: Fondo de Cultura Económica.

Foucault, M. (2008). Hermenéutica del sujeto (2. ${ }^{a}$ reimpresión). Buenos Aires: Fondo de Cultura Económica.

Merizalde, M. (2012, enero-diciembre). La escuela y la guerra, las memorias entre el miedo y el silencio. Praxis Pedagógica, 13, 90-103.

Pál Pelbart, P. (2006). Biopolítica y contranihilismo. Nómadas, 26, 8-19.

Restrepo Yusti, M. (1999). Escuela y desplazamiento. Colombia: Ministerio de Educación Nacional.

Romero Medina, F. A. (2013). Conflicto armado, escuela, derechos humanos y DIH en Colombia. Análisis Político, 26 (77), 57-84. Recuperado de: http://www.scielo.org.co/scielo.php?script=sci_arttext\&pid= S0121-47052013000100003\&lng=en\&tlng=es.

Sánchez A., R. (2007). Alcances y límites de los conceptos biopolítica y biopoder en Michel Foucault. En R. Sánchez A. (ed.). Biopolítica y formas de vida. Bogotá: Pontificia Universidad Javeriana.

Suárez, H. (2014). Si matan un maestro más serán 1000. En Las Dos Orillas, revista electrónica. Recuperado de: http://www.las2orillas.co/si-matanun-maestro-mas-seran-1000/. 